\title{
Bio-Optimized Curcuma as Adjuvant Therapy of Osteoarthritis and Its Influence on Gene Regulation (Sirt1), Metabolic Inflammation and Associated Symptoms.
}

\author{
Sara Bianchi ${ }^{1,3 *}$, Maurizio Salamone ${ }^{2}$, Greet Vanheule ${ }^{2}$, Mieke Van Den Driessche ${ }^{2}$, Ilaria Vignale ${ }^{3}$ and Luigi Vignale $^{3}$ \\ ${ }^{1}$ Department of Translational Research and New Technologies in Medicine and Surgery, Medical School - Pharmacology Section, University of \\ Pisa, Pisa, Italy. \\ ${ }^{2}$ Metagenics EU, Italy. \\ ${ }^{3}$ Studio Medico Vignale, Carrara MS, Italy.
}

*Corresponding author: Sara Bianchi, Department of Translational Research and New Technologies in Medicine and Surgery, Medical School - Pharmacology Section, University of Pisa, Pisa, Italy and Studio Medico Vignale, Carrara MS, Italy.

\section{Received date: May 03, 2021; Accepted date: May 12, 2021; Published date: May 25, 2021}

Citation: Sara Bianchi, Maurizio Salamone, Greet Vanheule, Mieke V D Driessche, Ilaria Vignale, et al (2021). Bio-Optimized Curcuma as Adjuvant Therapy of Osteoarthritis and Its Influence on Gene Regulation (Sirt1), Metabolic Inflammation and Associated Symptoms. J. Nutrition and Food Processing, 4(4); DOI:10.31579/2637-8914/052

Copyright: () 2021 Sara Bianchi. This is an open access article distributed under the Creative Commons Attribution License, which permits unrestricted use, distribution, and reproduction in any medium, provided the original work is properly cited.

\begin{abstract}
Background and aim: Osteoarthritis $(\mathrm{OA})$ is a chronic degenerative disease characterized by an inflammatory state and significant oxidative stress. As curcuma is known for its anti-inflammatory and antioxidant activities, it could be used as alternative therapy next to or together with the standard treatment. This treatment consists of analgesics, steroids or non-steroidal anti-inflammatory drugs (NSAIDs) to reduce pain and inflammation related symptoms. The current study investigates the effect of bio-optimized curcuma on genetic (SIRT1) and metabolic regulation of inflammation and associated symptomatology in patients with osteoarthritis.
\end{abstract}

Materials and methods: In the in vitro study, Hela human cells were seeded in 12-well plates, incubated with curcuma at different concentrations and incubated for 3, 6 and 24 hours. The targeted protein expression/phosphorylation was evaluated by immunoblotting, while cytotoxicity tests were performed by CellTiter-Blue Cell Viability Assay. In the in vivo study, a total of 33 patients were recruited and divided into 3 subgroups based on the treatment: standard treatment (ST), ST + curcuma and ST + curcuma + Vitamin D (2000UI). The health status (SF36) and osteoarthritis index (WOMAC score) were analyzed at $0 ; 1,5$ and 3 months with blood sampling at 0 and 3 months for the evaluation of inflammation markers, $25(\mathrm{OH})$ VitD and SIRT1.

Results: in vitro data showed no statistically significant decrease $(\mathrm{p}>0.05)$ in the number of viable cells. The expression of SIRT1 and the activation of AMP activated protein kinase (AMPK) were significantly increased in all experimental groups compared with the control group $(\mathrm{p}<0.001)$. A significant increase in $25(\mathrm{OH})$ VitD values in the ST + curcuma + VitD group $(\mathrm{p}<0.007)$ and in SIRT1 in all groups taking curcuma $(\mathrm{p}<0.001)$ was shown. Also for IL-1 ( $\mathrm{p}=0.031)$, IL$6(\mathrm{p}=0.031)$ and IFN- $\gamma(\mathrm{p}=0.013)$, all groups taking curcuma showed significantly lower inflammatory markers with no added value of vitamin $\mathrm{D}$.

Conclusions: Curcuma as an adjuvant to ST leads to a positive modulation of the SIRT1 pathway, a significant decline of blood inflammatory markers and a better osteoarthritis outcome.

- Osteoarthritis, a chronic degenerative disease characterized by an inflammatory state and significant oxidative stress

- Curcuma as alternative therapy next to or together with the standard treatment

- Effect of bio-optimized curcuma on genetic and metabolic regulation of inflammation

- Positive modulation of SIRT1 pathway, decline of blood inflammatory markers and a better OA outcome

Key words: bio-optimized curcuma; osteoarthritis; inflammation; SIRT1; Womac Score; NAMPT; metalloproteinases; proinflammatory cytokines; PMSF; synoviocytes; Curcudyn 


\section{Introduction}

Osteoarthritis (OA) is a multifactorial degenerative joint disease evolving over time with no resolutive cure to date [1-3]. OA is characterized by an inflammatory state and significant oxidative stress resulting in pain, stiffness, swelling and loss of mobility [4]. The world prevalence of knee osteoarthritis increased with $26.6 \%$ from 1990 to 2010 and about $12 \%$ of the general population is suffering from OA [4-5].

In OA, synoviocytes and synovial macrophages produce inflammatory mediators including prostaglandins, reactive oxygen species and proinflammatory cytokines such as Interleukin (IL)-6, Tumor Necrosis Factor (TNF)- $\alpha$. [6,7]. These proinflammatory cytokines stimulate articular chondrocytes and synoviocytes to produce matrix-degrading enzymes such as Matrix metalloproteinases (MMPs) and proinflammatory enzymes such as Cyclooxygenase-2 (Cox-2), leading to a vicious circle and cartilage extracellular matrix destruction and degeneration [8]. The whole process enhances the apoptosis of chondrocytes which is further stimulated by the production of reactive oxygen species [6-8].

The current treatment focusses mainly on the reduction of pain and inflammation with analgesics, steroids and Non-Steroidal AntiInflammatory Drugs (NSAIDs) [2,9]. However, they fail to modify the disease progression in term of prevention and chondroprotection and their long-term use cannot be sustained due to inadequate pain relief, immune disturbances and serious gastrointestinal, renal and cardiovascular adverse events $[1,2,4,9]$. Therefore, herbal therapies with antiinflammatory properties have been investigated due to minimal side effects. In addition, the impaired balance between anabolic and catabolic mechanisms leading to articular damage, can also be influenced by dietary compounds like nutraceuticals, food products providing health and medical benefits such as Curcuma longa and Zingiber Officinale [1, 1014].

Curcuma is a yellow pigment isolated from the rhizomes of Curcuma Longa $[1,15,16]$ and is composed of demethoxycurcumin, bisdemethoxycurcumin, 5'-methoxycurcumin and dihydrocurcumin, collectively named curcuminoids $[15,16]$. Curcuminoids modify NF-kB signaling, proinflammatory cytokine production such as interleukins and phospholipase A2, COX-2 and 5-LOX activities resulting in anti-oxidant, anti-inflammatory and analgesic properties [10-18]. Curcumin itself is also a free radical scavenger and increases the binding of different molecules to the antioxidant response element, increasing the total antioxidant properties $[16,18]$. In addition, Curcumin might also be able to reverse the imbalance between anabolic and catabolic factors occurring in OA joint tissues by reducing the catabolic part [17]. In vitro studies on chondrocytes have confirmed the anti-inflammatory properties but have also shown anti-catabolic properties by the inhibition of MMP-3 synthesis resulting in decreased chondrolysis and decreased breakdown of extracellular matrix. Also anti-apoptotic activities on chondrocytes and growth-inhibitory and pro-apoptotic effects on synovial adherent cells, the main source of inflammatory mediators of cartilage degradation, have been confirmed $[19,20]$. Belcaro et al. showed significant improvement in OA symptoms, a three-fold increase in walking distance and a significant decrease in inflammatory markers after administration of a curcumin-phosphatidylcholine complex [21]. Kuptniratsaikul et al. showed that curcuma for knee OA treatment was as effective as ibuprofen but with fewer adverse events $[13,14]$.

Ginger has been an important ingredient in Asian medicine, especially for pain relief in musculoskeletal diseases $[1,22,23]$. Especially the gingerol and zingerone components have anti-inflammatory and anti-oxidant effects and are working synergistically with curcumin [24, 25]. Mozaffari-Khosravi et al. showed the inhibition of COX-2 and a decline in proinflammatory cytokines, TNF- $\alpha$ and IL- $1 \beta$ modulating NF-kB activities after the intake of ginger powder in patients with knee OA [25], another case study has shown relief of OA symptoms after ginger therapy [26]. In general, ginger reduces inflammation, pain and disability in OA without any side effects [22-25].

Regarding vitamins, Vitamin D is needed for normal bone metabolism as suboptimal levels may have an adverse effect on calcium metabolism, osteoblastic activity, matrix ossification and bone density [27, 28]. Supplementation with Vitamin D led to a lower disability of knee OA patients [27] and there might even be evidence that Vitamin D results in lower skeletomuscular pain [29]. In addition, Vitamin C is mostly known for its antioxidant function neutralizing the Reactive Oxygen species generated by cells within the joints and playing a role in OA [30]. Additionally, Vitamin $\mathrm{C}$ is also critical to bone health, as electron donor in the synthesis of type II collagen resulting in decreased cartilage degradation $[30,31]$

Herein, we investigate the effect of bio-optimized curcuma on genetic (SIRT1) and metabolic regulation of inflammation and associated symptomatology in patients with osteoarthritis.

\section{Materials and Methods}

\subsection{In vitro study}

\subsubsection{Chemicals and antibodies}

Cell culture reagents were purchased from Lonza (Basel, Switzerland) and Gibco-BRL (Grand Island, NY). General laboratory chemicals were purchased from Sigma-Aldrich (St. Louis, MO, USA) and Carlo Erba (Milano, Italy).

Ripa Lysis Buffer System, Protease Inhibitor Cocktail, Phosphatase Inhibitor Cocktail C, phenyl-methane-sulfonyl-fluoride (PMSF), Sodium Orthovanadate and Sodium Pyrophosphate were obtained from Santa Cruz Biotechnology (California, USA). CellTiter-Blue Cell Viability Assay was purchased from Promega (Madison, USA).

Reagents, protein markers and membrane for Western Blot were from Biorad Laboratories (California, USA), Rabbit polyclonal antibody against SIRT1 (H-300, sc-15404), mouse monoclonal antibody against Actin (C-2, sc-8432), goat anti-rabbit IgG-HRP antibody (sc-2004) and goat anti-mouse IgG-HRP antibody (sc-2005) were purchased from Santa Cruz Biotechnology (California, USA).

Rabbit polyclonal antibody against Phosho-AMPKa (Thr172, \#2531), rabbit polyclonal antibody against Total AMPKa (\#2532) were from Cell Signaling Technologies (Massachussets, USA). Luminata Crescendo Western HRP Substrate for chemiluminescent detection of bands were from Millipore (Massachussets, USA).

\subsubsection{Cell Culture}

Human Cervical Carcinoma cells (HeLa) were cultured in DMEM supplemented with $10 \%(\mathrm{v} / \mathrm{v})$ fetal bovine serum, $1 \%$ antibiotics (penicillin and streptomycin) and $1 \% \mathrm{~L}$-glutamine at $37^{\circ} \mathrm{C}$ in a humidified air atmosphere with $5 \%(\mathrm{v} / \mathrm{v}) \mathrm{CO} 2$.

The cells were grown to a confluence of $80 \%$, were then trypsinized, counted with a Burker chamber and plated (100.000 cells / well). The day of the experiment, the cells were treated with the substances at the different concentrations considered and left in incubation for 3, 6 and 24 hours.

At the end of the incubation periods, the cells were washed with PBS (pH 7.4) and collected in a $1.5 \mathrm{ml}$ microtube with $0.130 \mathrm{ml}$ of lysis buffer (RIPA buffer) containing a protease inhibitor cocktail, a phosphatase inhibitor cocktail, PMSF, sodium orthovanadate and sodium pyrophosphate. 
They were lysed by three freezing-thawing cycles after which the supernatants were recovered.

A part of the lysate was used to quantify the protein content with the spectrophotometer (Thermo Scientific Multiskan FC, Thermo Scientific), using a commercial kit based on the Bradford assay. The final concentrations are expressed in $\mu \mathrm{g} / \mu \mathrm{l}$.

\subsubsection{CellTiter-Blue Cell Viability Assay}

The CellTiter-Blue Cell Viability Assay provides a homogeneous fluorometric method for estimating the number of viable cells present in multi-well plates. It uses resazurin, an indicator dye, to measure the metabolic capacity of cells to determine cell viability. As only viable cells reduce resazurin into resorufin, which is highly fluorescent, fluorescence is linked to cell viability.

Cell viability was carried out in 96-well plates and after obtaining the desired density, they were treated with the substances to be analyzed and appropriately diluted in DMSO.

5000 cells/well were plated, in a final volume of $100 \mu \mathrm{L}$ per well. The cells were treated with above-mentioned substances and incubated for 3, 6 and 24 hours.

Subsequently, the reagent $(20 \mu \mathrm{L} /$ well $)$ was added and the plate was incubated in the dark for 4 hours at $37^{\circ} \mathrm{C}$. Finally, the fluorescence $(560 \mathrm{Ex} / 590 \mathrm{Em})$ was detected using Cytofluor 2300 (Millipore Corporation. USA). The produced fluorescence is directly proportional to the number of viable cells and, these values were compared and expressed with respect to control (cells without treatment).

\subsection{In vivo study}

\subsubsection{Study design}

This open label, randomized study included 33 patients from Italy enrolled between January and March 2016.

The main inclusion criteria were: age between 24 and 82 years with shoulder, hip or knee OA. The main exclusion criteria were dementia, inability to give informed consent, pregnancy or lactation, planned knee replacement surgery, allergy or contraindication to curcumin, and joint diseases other than OA. The use of any intra-articular injection in the target knee in the last 3 months, the use of symptomatic slow-acting drugs in OA (SYSADOA) in the last month, oral corticotherapy $\geq 5 \mathrm{mg} /$ day in the last 3 months, the use of products containing curcuminoids extract in the last 3 months, was also specifically forbidden by the study protocol. All patients were instructed to not change the dietary habits nor medication.

The study was conducted in conformity with principles stated in the World Medical Association Declaration of Helsinki regarding on ethical conduct of research involving human subjects and was approved by Human Ethic Committee. All patients gave their written informed consent to participate.

\subsubsection{Treatment assignment}

The bio-optimized curcuma evaluated in this study was approved by the Competent Authorities and commercialized under the brand name Curcudyn ${ }^{\circledR}$ (Metagenics). Two capsules of CurcuDyn ${ }^{\circledR}$ contain $100 \mathrm{mg}$ active curcumin.

Patients $(n=33)$ were randomly assigned to one of the following three groups (Table 1): Control group receiving the standard treatment (ST), CurcuDyn group receiving standard treatment and curcuma for 3 months (ST+CurcuDyn) and the CurcuDyn + vitamin D (Vit D) group receiving standard treatment and curcuma for 1.5 months and the standard treatment, curcuma and vitamin D (Metagenics, 2000 UI) for the next 1.5 months.

The standard treatment consists of a hyaluronic acid in combination with corticosteroid injection performed for all patients at $\mathrm{T} 0$ (trial start), while Curcudyn ${ }^{\circledR}$ capsules were taken twice daily with water, once at breakfast and once at dinner for 15 days, and then once daily until the end of the study (3 months). Two capsules contain $105 \mathrm{mg}$ of turmeric rhizome extract (Curcuma longa L.), $16 \mathrm{mg}$ of Vitamina C (ascorbic acid), $5 \mu \mathrm{g}$ of Vitamin D (cholecalciferol) and $20 \mathrm{mg}$ of Gember extract (Zingiber officinalis).

The trial lasted three months with the following observational intervals: $0,1.5$ and 3 months.

For the further analysis, subjects with two locations of arthroses were assigned to a single location. Thus, in the control group, the subject with should/hip was assigned to hip and the subject with hip/knee was assigned to the knee group. In the Curcudyn + Vit D group, the subject with shoulder/knee was assigned to the shoulder group.

\subsubsection{Parameters evaluated}

\subsubsection{Short Form (SF36) Health Survey}

The Short Form (36) Health Survey, better known as SF36, is a set of generic, coherent, and easily administered quality-of-life measure. It is used to indicate the health status of particular populations, to help with service planning and to measure the impact of clinical and social interventions. SF36 consists of eight scaled scores, which are the weighted sums of the questions in their section. Each scale is directly transformed into a 0-100 scale on the assumption that each question carries equal weight.

\subsubsection{WOMAC SCORE}

The Western Onstario and McMaster Universities Arthritis Index (Womac) Score is widely used in the evaluation of Hip and Knee Osteoarthritis. It is a self-administered questionnaire consisting of 24 items divided into 3 subscales: pain ( 5 items), stiffness ( 2 items) and physical function (17 items). The WOMAC takes approximately 12 minutes to complete, and can be taken on paper, over the telephone or computer. Both the computerized and the mobile versions of the test have been found to be comparable to the paper form, with no significant difference.

The test questions are scored on a scale of $0-4$, which correspond to: None (0), Mild (1), Moderate (2), Severe (3), and Extreme (4). The scores for each subscale are summed up, with a possible score range of $0-20$ for Pain, 0-8 for Stiffness, and 0-68 for Physical Function. Usually a sum of the scores for all three subscales gives a total WOMAC score, however there are other methods that have been used to combine scores. Higher scores on the WOMAC indicate worse pain, stiffness, and functional limitations.

\subsubsection{Blood samples}

Blood samples were taken after an over-night fasting status, kept on ice and centrifuged at $2500 \mathrm{~g}, 4^{\circ} \mathrm{C}$ for $10 \mathrm{~min}$. Then, the serum was collected and stored at $-80^{\circ} \mathrm{C}$ until assayed.

The evaluation of IL-1, IL-6, IFN-y, TNF- $\alpha$ and SIRT1 was performed by a specific human Elisa test (Emelca, The Netherlands), while the remaining markers were measured with classical clinical biochemistry methods.

Both the SF36 and WOMAC SCORE were evaluated at T0, T1.5 and T3, while blood samples were collected at 0 and 3 months for the evaluation 
of inflammation markers (VES, gamma-GT, PCR, AST, ALT, IL-1, IL6, IFN-y, TNF- $\alpha), 25(\mathrm{OH})$ VitD and SIRT1.

At the time of enrolment, all participants shared detailed information about their clinical history and life style habits.

\subsection{Statistical analyses}

Descriptive statistics of all demographic, baseline variables and study parameters were provided overall. Continuous data were summarized by their mean, standard deviation, median, minimum and maximum. All data are expressed as mean \pm Standard Error of the Mean (SEM).

In the in vitro study, the effect of increasing concentrations of curcuma and of different exposure times on the average viability (Hela cells), SIRT1 expression and AMPK activation was investigated with a two-way ANOVA. The assumptions underlying the model were assessed with diagnostic plots (QQ-plot of residuals, residuals versus fitted plots).

For the continuous parameters, mean changes (difference between T0 and T3) between the different treatment groups were explored using a one- way analysis of variance (one-way ANOVA). The normality of the residuals was investigated with a qq-plot. The homogeneity of variances was tested with the Levene's test. In case of a significant ANOVA, the multiple comparison Scheffe's procedure was used to investigate which of the treatments were different. A value of $\mathrm{p}<0.05$ was considered statistically significant. All analyses were performed in $\mathrm{R}$ version 3.2.0. (R Core Team, 2018).

\section{Results}

\subsection{In vitro results}

\subsubsection{CellTiter-Blue Cell Viability Assay (Promega) results.}

Hela cells were treated with $0,2.5,5,7.5$ and $10 \mu \mathrm{l}$ of curcuma over $3 \mathrm{~h}$, $6 \mathrm{~h}$ and $24 \mathrm{~h}$ to determine the viability by cell count. The effect of increasing concentrations of curcuma on the viability of Hela cells is presented in Figure 1. The viability was not affected up to a concentration of $10 \mu \mathrm{L}$ of curcuma and the viability remained high after a $3 \mathrm{~h}$ $($ mean $=91.6 \%), 6 \mathrm{~h}($ mean $=89.3 \%)$ and $24 \mathrm{~h}($ mean $=90.9 \%)$ exposure.

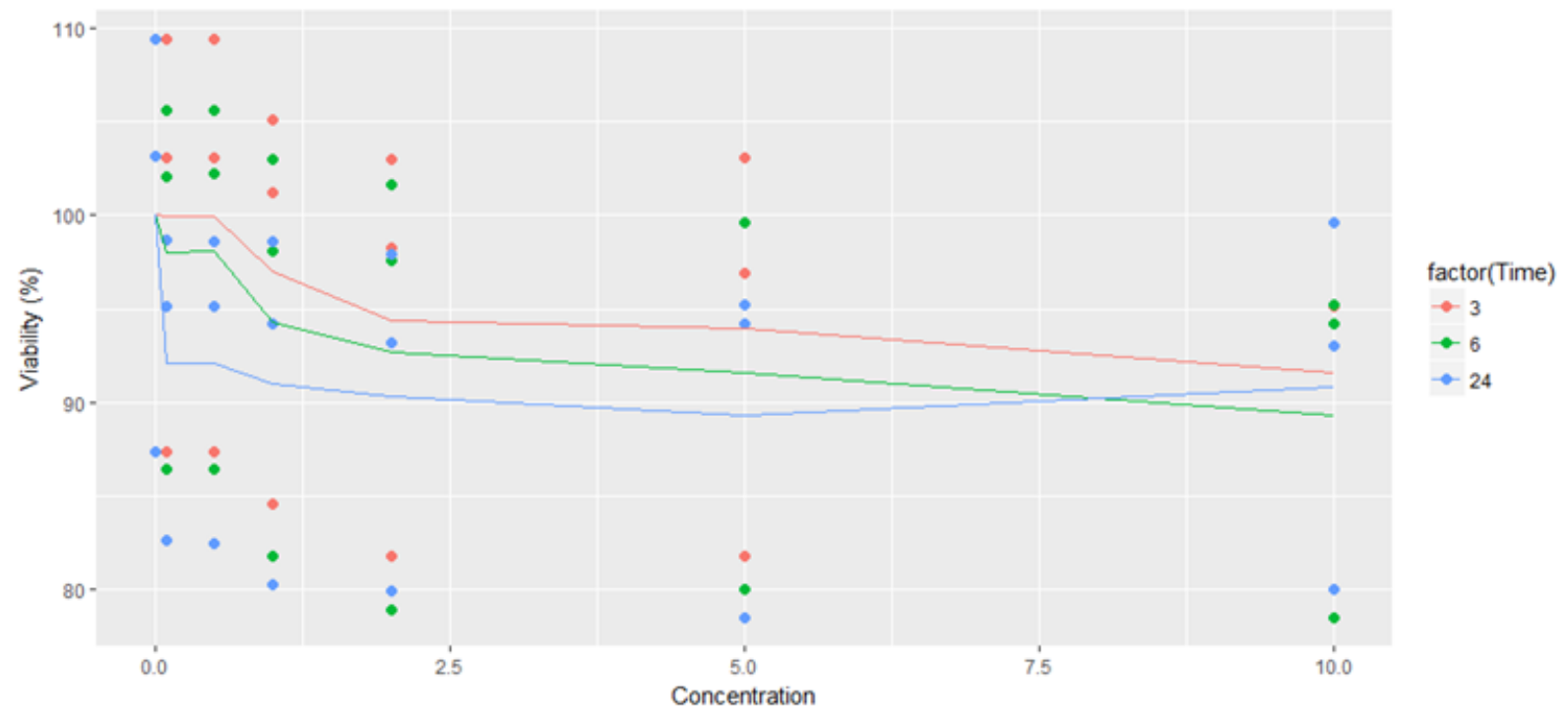

Figure 1. Effect of Curcudyn concentration on the viability of Hela cells.

The line corresponds with the average of 3 samples, the dots with the individual viability. The time (3, 6 and 24 hours) corresponds with the exposure time for the different concentrations $(0,2.5,5,7.5$ and $10 \mu 1)$.

3.1.2 The expression of SIRT1 was significantly increased in all experimental groups compared with the control group.

The effect of increasing concentrations of curcuma and of different exposure times (3h, 6h, and 24h) on the expression of SIRT1 (Western
Blot analysis of the lysates of the cells incubated with the tested compound, Figure 2A-B) was investigated with a two-way ANOVA. Increasing concentrations of Curcudyn did result in a statistically significantly higher expression of SIRT1 in comparison with the control treatment $(\mathrm{F}(2,32)=16.6, \mathrm{p}<0.001)$ but the expression was not influenced by the exposure time $(\mathrm{F}(2,32)=0.363, \mathrm{p}=0.699)$. 


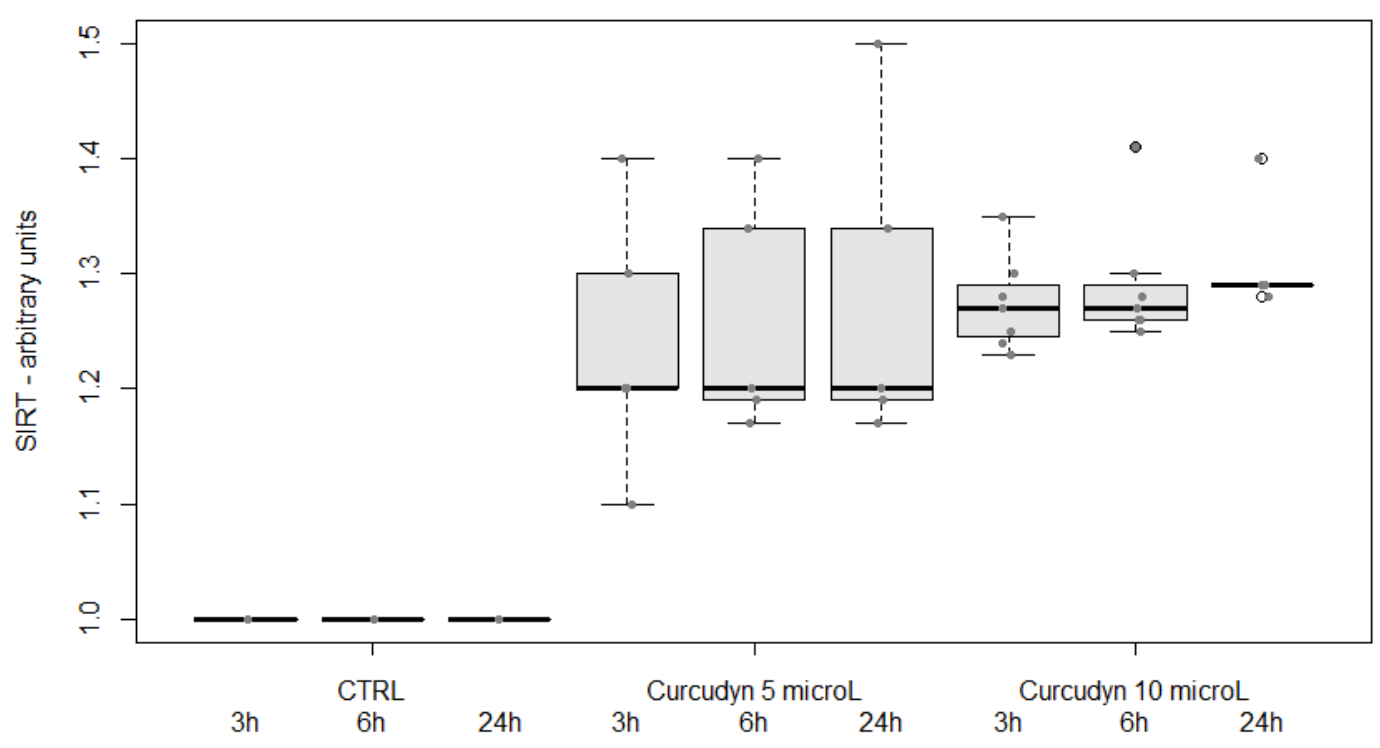

The time corresponds with the exposure time for the different concentrations

SIRT1

$\beta$-actina
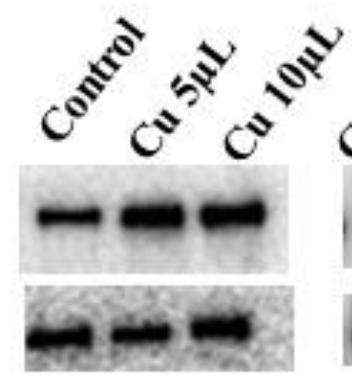

3h

$6 \mathrm{~h}$

24h

Figure 2B. Representative blots of experiments in which SIRT1 expression (SIRT1) was normalized with $\beta$-actin housekeeping gene ( $\beta$-Act). The statistical significance was ***p $<0.001$ vs. CT

The next phase verified if curcuma, proven to be a SIRT1 activator, was also able to modulate and interact with the AMPK pathway. The Western Blot analysis showed that the activation of AMPK is significantly increased in all experimental groups compared with the control group, at all concentrations and all experimental times $(\mathrm{p}<0.001)$ (Figure 3 ) 


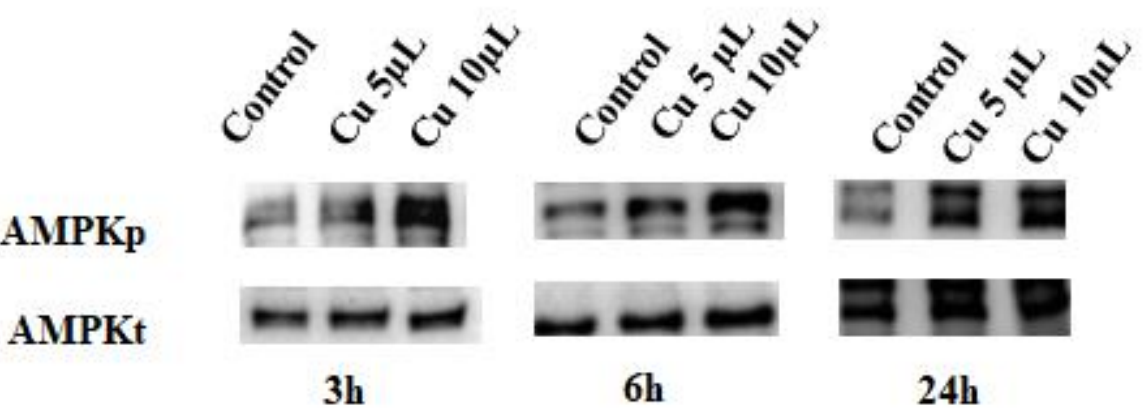

Figure 3. Representative blots of experiments in which phosphor-AMPK (AMPK-p) was normalized with total AMPK (AMPK-t). The statistical significance was $* * * p<0.001$ vs. $C T$

\subsection{In vivo results}

Table 1.

\begin{tabular}{|c|c|c|c|}
\hline Variable & Control $(\mathrm{N}=7)$ & Curcudyn ( $N=16)$ & Curcudyn + Vit D ( $N=10)$ \\
\hline \multicolumn{4}{|l|}{ Age (Years) } \\
\hline mean & 55.1 & 58.9 & 57.5 \\
\hline Min-Med-Max & $45-55-62$ & $24-57.5-75$ & $40-54.5-82$ \\
\hline \multicolumn{4}{|c|}{ Location of arthroses } \\
\hline shoulder & 1 (s houlder/hip) & 5 & 3 ( $1 / 3 \mathrm{~s}$ houlder $/ \mathrm{k}$ nee $)$ \\
\hline Hip & 3 (1/3 hip/k nee) & 6 & 4 \\
\hline Knee & 3 & 5 & 3 \\
\hline
\end{tabular}

Table 1. Demographic characteristics

\subsubsection{SF 36 results}

The distribution of the responses to the SF36 questionnaire was reported in specific tables and appropriately analyzed (tables not shown).

Briefly, the results show benefits in 76 percent of patients, more precisely 25 patients out of 33 total. These 25 patients all belong to groups treated with bio-optimized curcumin. On the other hand, results show no benefit in 24 percent or 8 out of 33 patients. Of these, 7 were in the control groupor patients only treated with the SD. To conclude, the assessment of the SF36 showed that only one patient treated with Curcudyn didn't report any benefit.

\subsubsection{WOMAC Score}

The analysis of the WOMAC Score showed a different response in knee and hip OA patients. In further analysis, the comparison of change in WOMAC will be performed for knee and hip separately (Figure 4) 


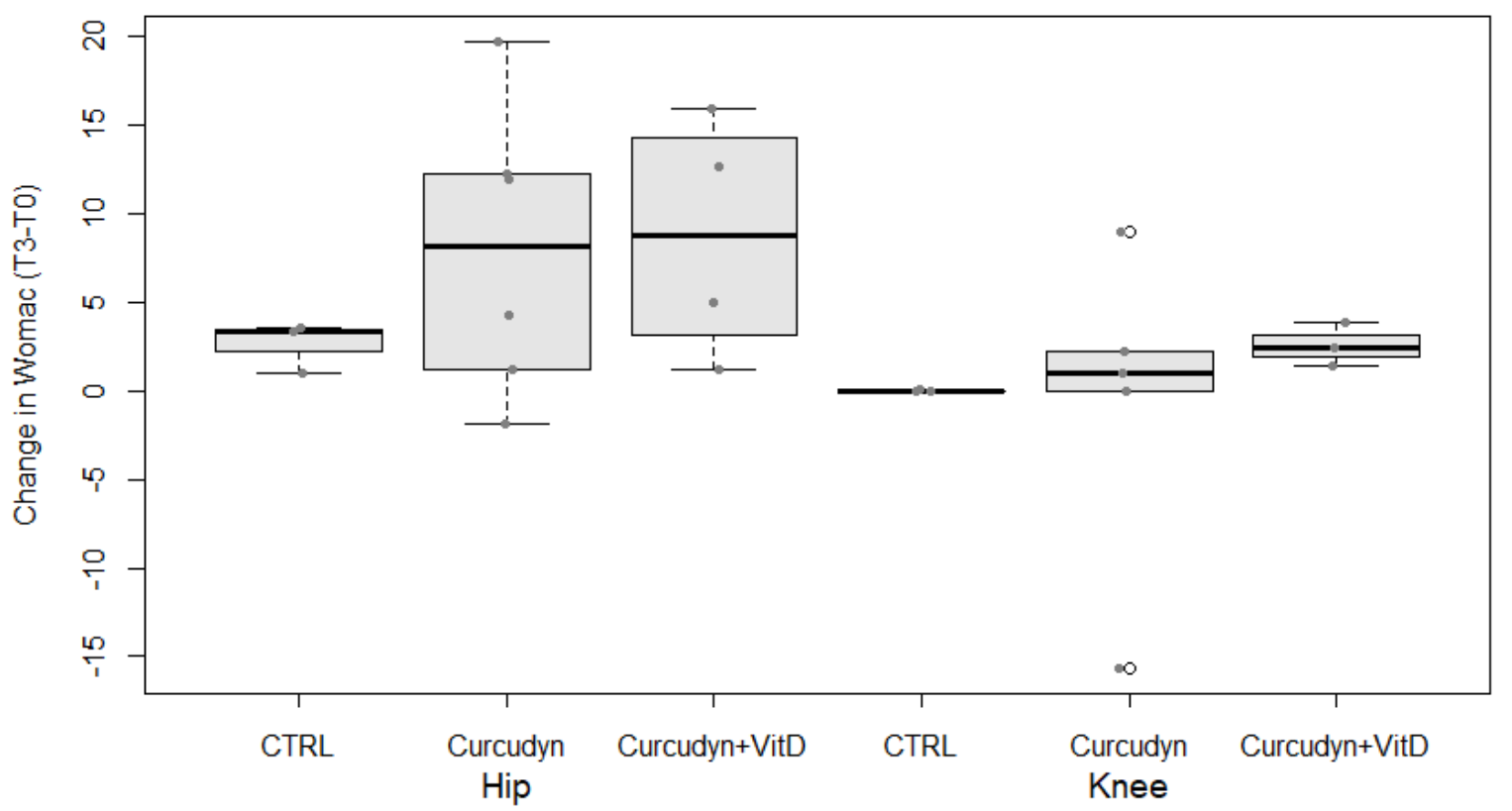

Figure 4. Boxplot for change in Womac, the dots correspond with the individual changes

Regarding hip OA, there was no evidence of a statistically significant difference between the treatment groups as determined by a one-way ANOVA $(\mathrm{F}(2,10)=0.777, \mathrm{p}=0.486$, Figure 3$)$. The mean change of
WOMAC for hip $\mathrm{OA}$ at 3-months follow-up from baseline were comparable between the different treatments (Table 2)

\begin{tabular}{|l|l|l|l|l|l|}
\hline Treatment & N & Mean & SD & Min & Max \\
\hline Control & 3 & 2.67 & 1.45 & 1.00 & 3.62 \\
\hline Curcudyn & 6 & 7.94 & 8.12 & -1.89 & 19.73 \\
\hline Curcudyn+VitD & 4 & 8.73 & 6.79 & 1.25 & 16.00 \\
\hline
\end{tabular}

Table 2. Summary statistics for changes in Womac for Hip OA patients (T3-TO)

Also for knee OA, there was no evidence of a statistically significant difference between the treatment groups as determined by a one-way ANOVA $(F(2,8)=0.245, p=0.788$, Figure 4$)$. The mean change of
WOMAC for the knee OA patients at 3-months follow-up from baseline were comparable between the different treatments (Table 3).

\begin{tabular}{|l|l|l|l|ll|}
\hline Treatment & N & Mean & SD & Min & Max \\
\hline Control & 3 & 0.01 & 0.02 & 0.00 & 0.04 \\
\hline Curcudyn & 5 & -0.67 & 9.07 & -15.64 & 8.98 \\
\hline Curcudyn+VitD & 3 & 2.58 & 1.23 & 1.40 & 3.85 \\
\hline
\end{tabular}

Table 3. Summary statistics for changes in Womac for knee OA (T3-TO)

\subsubsection{Blood inflammation markers}

3.2.4.1 No statistically significant difference for VES, gamma GT, PCR were found
Figure 5A-C shows no evidence of a statistically significant difference between the treatment groups as determined by a one-way ANOVA both for $\operatorname{VES}\left(\mathrm{F}_{(2,30)}=1.73, \mathrm{p}=0.195\right)$, gamma $\operatorname{GT}\left(\mathrm{F}_{(2,30)}=0.998, \mathrm{p}=0.381\right)$ and PCR $\left(\mathrm{F}_{(2,30)}=0.159, \mathrm{p}=0.854\right)$, respectively. 


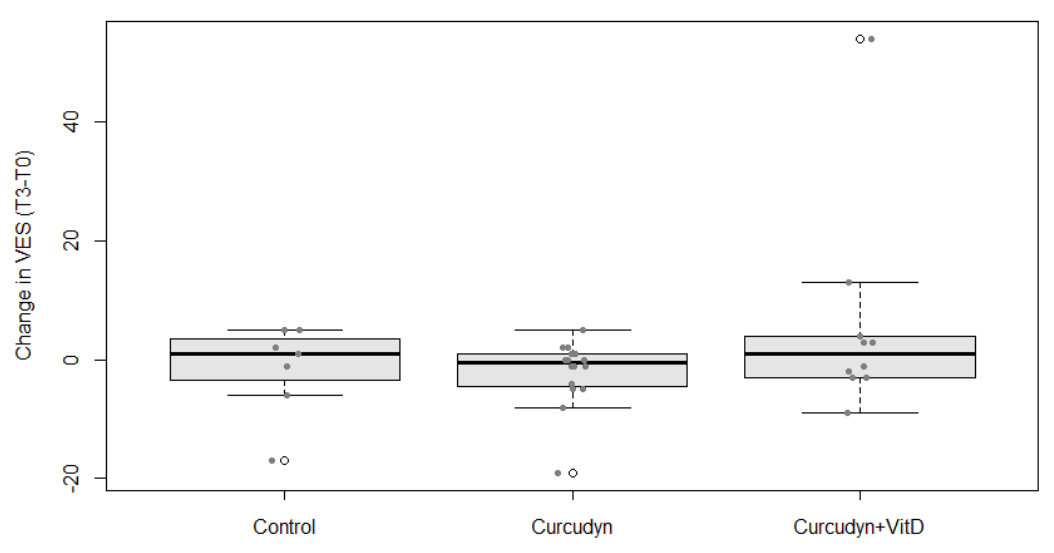

(Figure 5A)

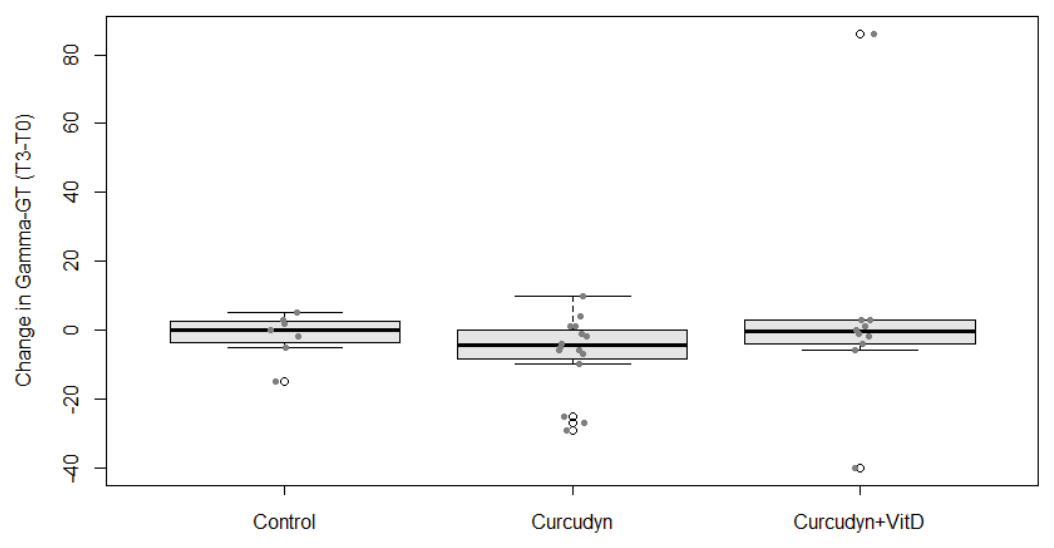

(Figure 5B)

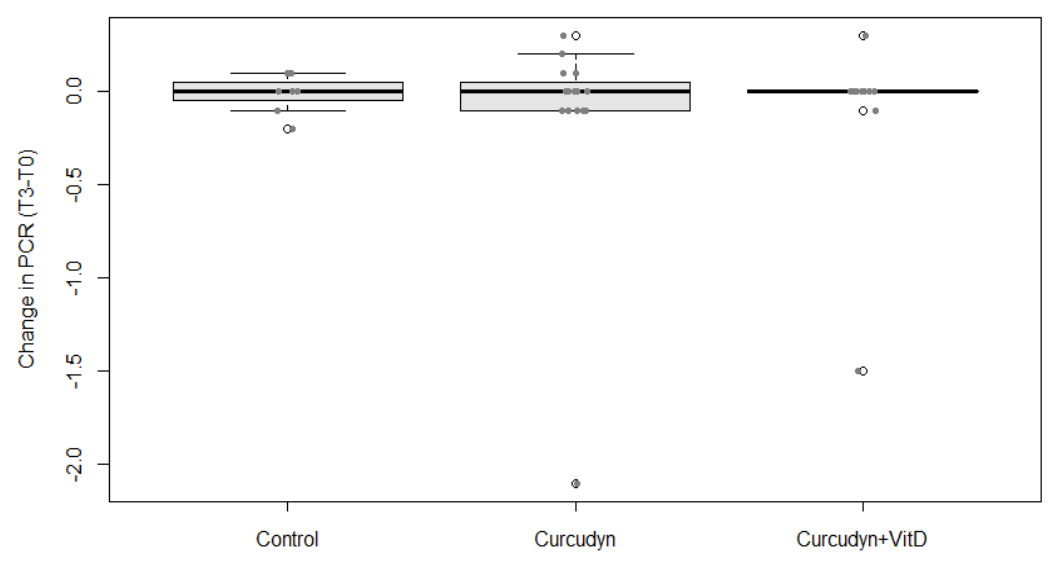

(Figure 5C)

Figure 5A-5C. Boxplot for change in blood inflammatory biomarkers, the dots correspond with the individual changes: (A) VES; (B) gamma GT; (C) PCR. 
The mean change of VES, gamma GT and PCR levels at 3-month followup from baseline were comparable between the different treatments (Tables not shown).

\subsubsection{Vitamin D levels were increased by Curcudyn treatment}

The vitamin D level analysis shows the presence of a statistically significant difference between the treatment groups as determined by a one-way $\operatorname{ANOVA}(\mathrm{F}(2,30)=3.31, \mathrm{p}=0.004$, Figure 6$)$.

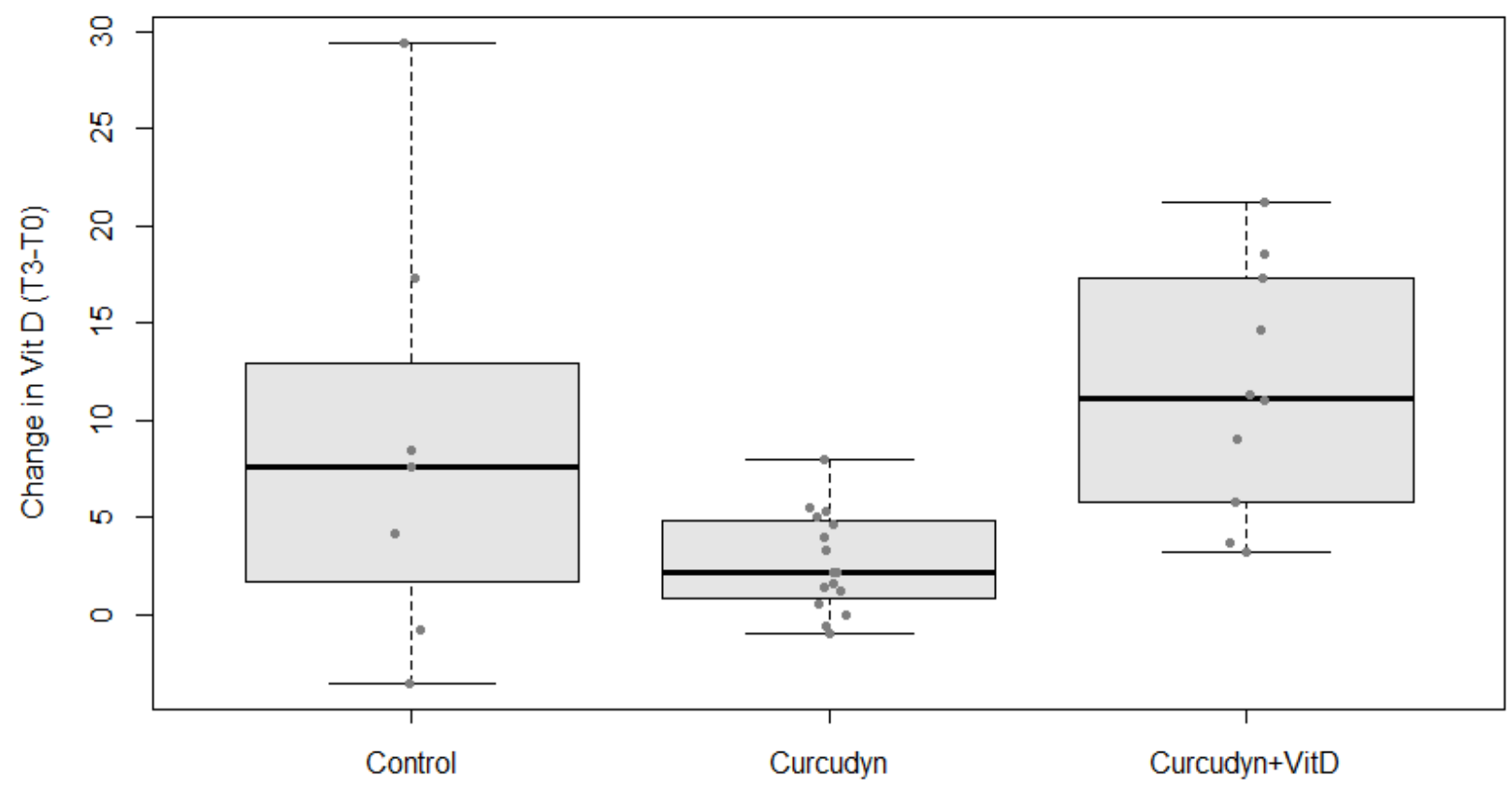

Figure 6. Boxplot for change in Vit D, the dots correspond with the individual changes

A Scheffe post-hoc test (Table 4) revealed that the increase in mean vitamin D levels was significantly higher after taking Curcudyn + Vit D (11.6 $\pm 6.3 \mathrm{~min}, \mathrm{p}=0.007)$ compared to Curcudyn $(2.7 \pm 2.5 \mathrm{~min})$. There was no statistically significant difference between the Control and Curcudyn group $(\mathrm{p}=.113)$ and between the Control and Curcudyn + Vit $\mathrm{D}$ group $(\mathrm{p}=.0 .708)$.

\begin{tabular}{|l|l|l|l|l|l|}
\hline Comparisons & Difference & Lower CI & Upper CI & p-value & $\begin{array}{l}\text { Scheffe } \\
\text { homogeneous } \\
\text { subsets }\end{array}$ \\
\hline Curcudyn vs Control & -6.2 & -13.7 & 1.2 & 0.113 & AB \\
\hline Curcudyn+VitD vs Control & 2.6 & -5.5 & 10.7 & 0.708 & A \\
\hline Curcudyn+VitD vsCurcudyn & 8.9 & 2.3 & 15.5 & 0.007 & B \\
\hline
\end{tabular}

Table 4. Posthoc multiple comparisons of means: Scheffe Test 95\% family-wise confidence level

\subsubsection{The tested cytokine levels significantly decrease after} Curcudyn treatment, except forTNFa.

The analysis of cytokine levels shows different responses. IL-1, IL-6 and IFN-y levels significantly decrease after Curcudyn treatment, in contrast to TNF $\alpha$ levels.
Regarding IL-1, there was a statistically significant difference between the treatment groups as determined by a one-way $\operatorname{ANOVA}(\mathrm{F}(2,30)=$ 4.78, p 0.016, Figure 7). 


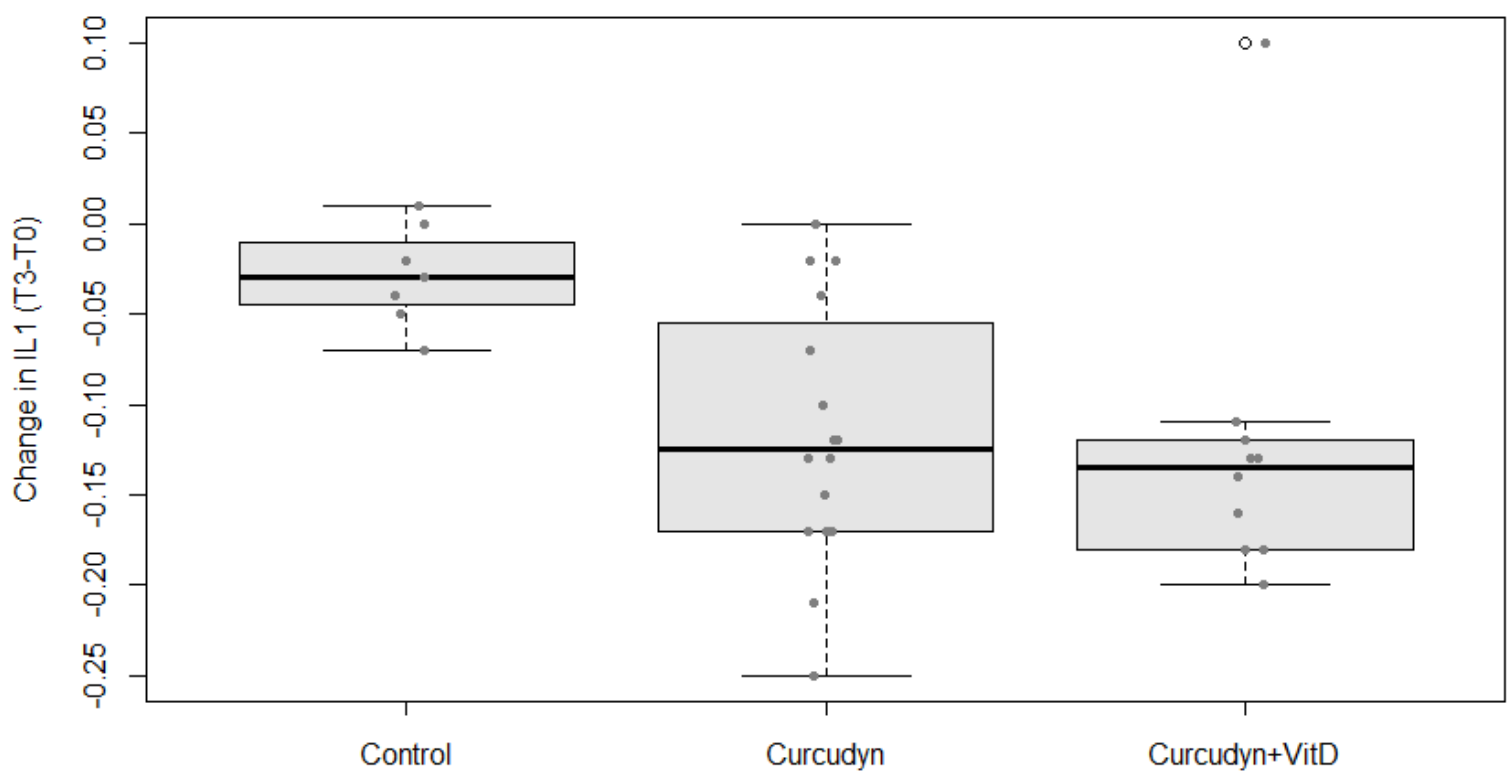

Figure 7. Boxplot for change in $I L-1$, the dots correspond with the individual changes

A Scheffe post-hoc test (Table 5) revealed that the decrease in mean IL-1 levels was significantly higher after taking Curcudyn $(-0.12 \pm 0.07 \mathrm{~min}$, $\mathrm{p}=0.031)$ and Curcudyn + Vit D $(-0.13 \pm 0.08 \mathrm{~min}, \mathrm{p}=0.030)$ compared to the Control group $(-0.03 \pm 0.03 \mathrm{~min})$. There was no significant difference between the Curcudyn and Curcudyn + Vit D group $(\mathrm{p}=$ 0.959).

\begin{tabular}{|l|l|l|l|l|}
\hline Comparisons & Difference & Lower CI & Upper CI & p-value \\
\hline Curcudyn vsControl & -0.09 & -0.17 & -0.01 & 0.031 \\
\hline Curcudyn+VitD vsControl & -0.10 & -0.18 & -0.01 & 0.030 \\
\hline Curcudyn+VitD vsCurcudyn & -0.01 & -0.08 & 0.06 & 0.959 \\
\hline
\end{tabular}

Table 5. Posthoc multiple comparisons of means: Scheffe Test $95 \%$ family-wise confidence level (IL-1)

Also for IL-6 and IFN- $\alpha$ levels was showed a statistically significant difference between the treatment groups as determined by one-way ANOVA (F $(2,30)=3.89, \mathrm{p} 0.032$ and $\mathrm{F}_{(2,30)}=7.43, \mathrm{p} 0.002$, Figure $\left.8 \mathrm{~A}-\mathrm{B}\right)$ 

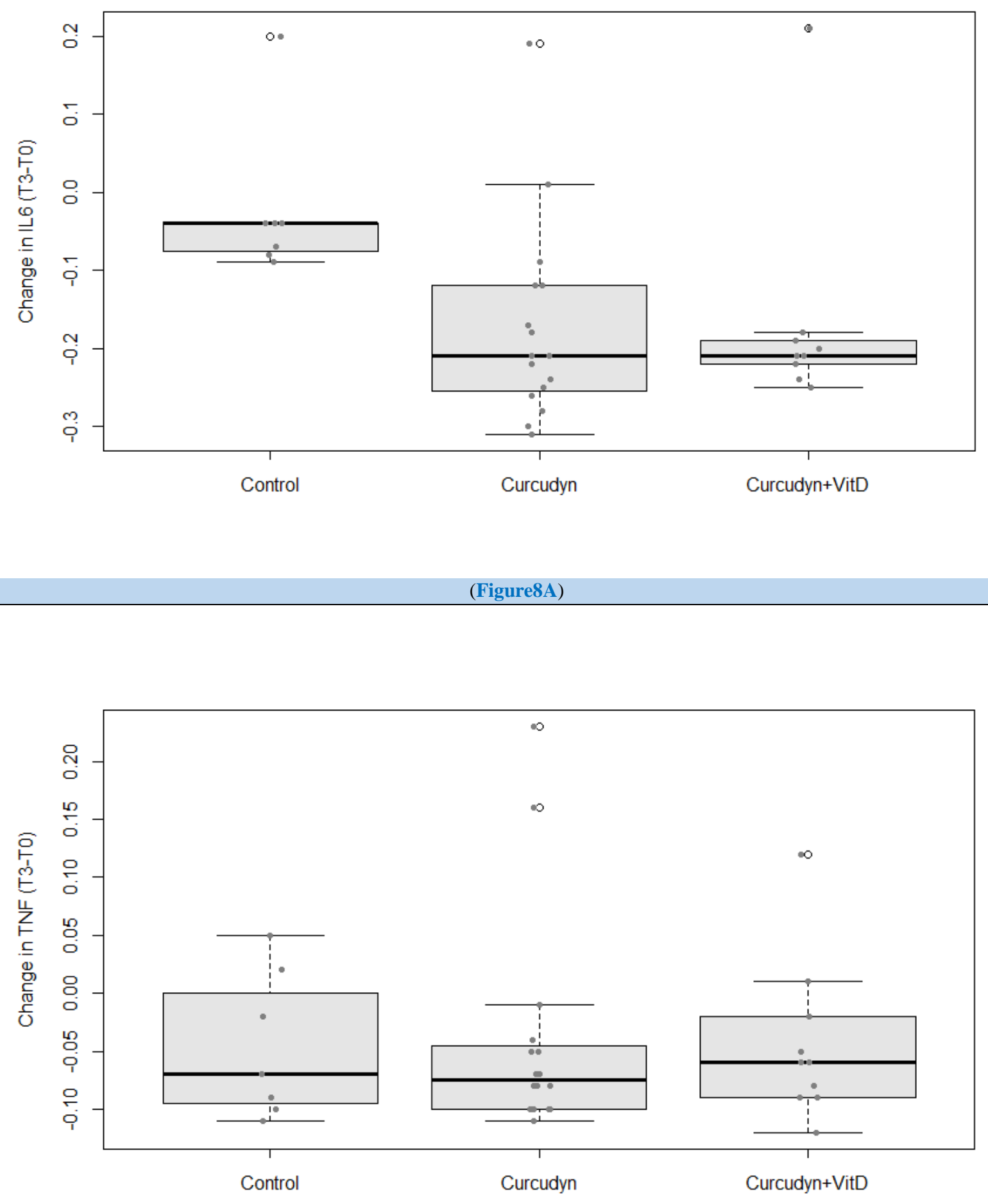

(Figure 8B)

Figure 8A-8B. Boxplot for change in cytokines levels, the dots correspond with the individual changes: (A) IL6; (B) IFN-y

A Scheffe post hoc test (data not shown) revealed that the decrease in mean IL6 and IFN-y levels were statistically significantly higher after taking Curcudyn $(-0.17 \pm 0.13 \mathrm{~min}, \mathrm{p}=0.031$ and $-0.13 \pm 0.08 \mathrm{~min}, \mathrm{p}=$ 0.013 , respectively). While for IL6 was borderline (not statistically 
different) the decrease in mean after Curcudyn + Vit $\mathrm{D}(-0.17 \pm 0.14 \mathrm{~min}$, $\mathrm{p}=0.073)$ compared to Control $(-0.02 \pm 0.10 \mathrm{~min})$, instead for INF-y was statistically significant too $(-0.16 \pm 0.07 \mathrm{~min}, \mathrm{p}=0.004$ vs $-0.03 \pm 0.05$ $\mathrm{min})$. There was no statistically significant difference between the Curcudyn and Curcudyn + Vit D groups both for IL6 $(\mathrm{p}=0.999)$ and for INF-y $(\mathrm{p}=0.635)$.
In contrary, the analysis of $\mathrm{TNF} \alpha$ levels evidenced no e statistically significant difference between the treatment groups as determined by a one-way ANOVA $\left(\mathrm{F}_{(2,30)}=0.011, \mathrm{p}=0.989\right.$, Figure 9).

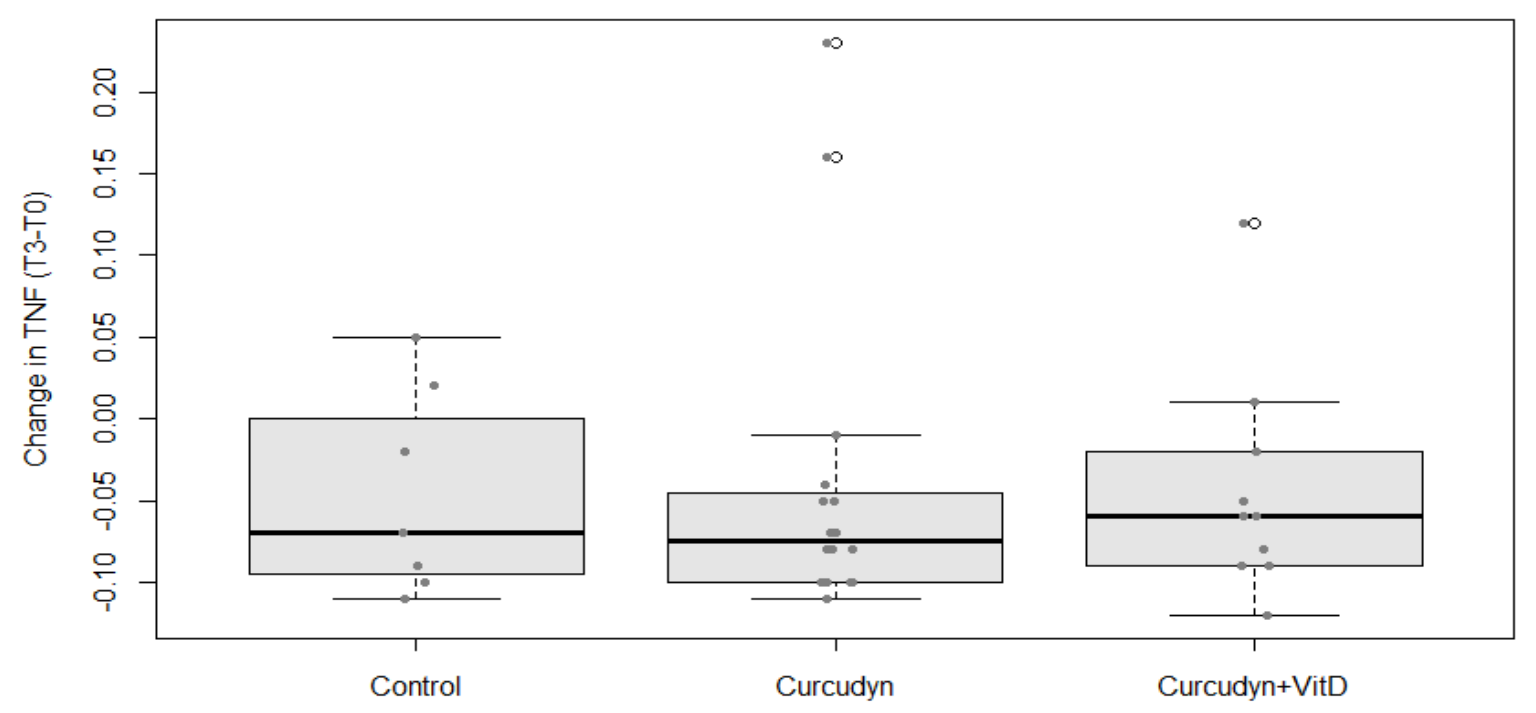

Figure 9. Boxplot for change in TNF $\alpha$, the dots correspond with the individual changes

3.2.4.4 SIRT 1 levels increased significantly after Curcudyn treatment.

Figure 10, shows the increase in SIRT1 levels after Curcudyn treatment.
Briefly, there was a statistically significant difference between the treatment groups as determined by a one-way $\operatorname{ANOVA}(\mathrm{F}(2,30)=17.7$, $\mathrm{p}<0.001)$.

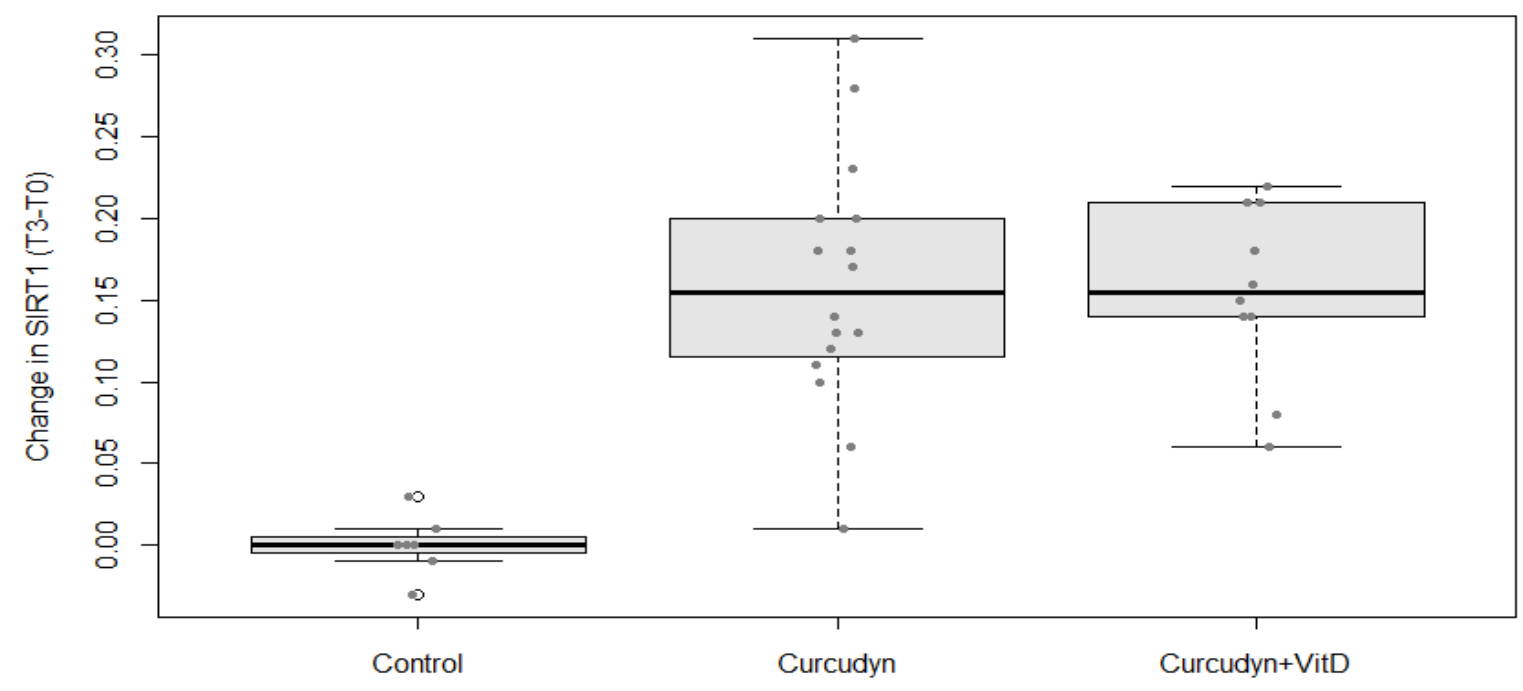


A Scheffe post-hoc test (Table 6) revealed that the increase in mean SIRT1 levels was significantly higher after taking Curcudyn (0.16 \pm 0.08 min, $\mathrm{p}$ $<0.001)$ and Curcudyn + Vit D $(0.16 \pm 0.05$ min, $\mathrm{p}=.007)$ compared to the Control group $(0.00 \pm 0.02$ min $)$. There was no statistically significant difference between the Curcudyn and Curcudyn + Vit D group $(\mathrm{p}=0.985)$.

\begin{tabular}{|l|l|l|l|l|}
\hline Comparisons & Difference & Lower CI & Upper CI & p-value \\
\hline Curcudyn vs Control & 0.16 & 0.09 & 0.23 & $<0.001$ \\
\hline Curcudyn+VitD vs Control & 0.16 & 0.08 & 0.23 & $<0.001$ \\
\hline Curcudyn+VitD vs Curcudyn & 0.00 & -0.07 & 0.06 & 0.985 \\
\hline
\end{tabular}

Table 6. Posthoc multiple comparisons of means: Scheffe Test 95\% family-wise confidence level (SIRT1)

\section{Discussion}

$\mathrm{OA}$ is a degenerative disease of the movable joints affecting mostly the elderly worldwide. It is characterized by local loss of cartilage, remodeling of adjacent bone, and bony overgrowth. It is the consequence of a maladaptive repair process of the cartilage due to overwhelming trauma. Its presentation is highly variable between individuals, with common features as joint pain, impaired movement, tenderness, crepitus, occasional effusion, and local inflammation [1-3]. Kraus et al. proposed that an altered inflammatory state is the underlying cause of OA, with mechanical stress as inducer [32]. More precisely, in OA, synoviocytes and synovial macrophages produce inflammatory mediators including prostaglandins, reactive oxygen species and proinflammatory cytokines such as IL-6, TNF- $\alpha$, etc. [6-8, 32]. These proinflammatory cytokines stimulate articular chondrocytes and synoviocytes to produce matrixdegrading enzymes such as MMPs and proinflammatory enzymes such as COX-2, leading to a vicious circle and cartilage extracellular matrix destruction and degeneration [6-8].

Currently, the available pharmacological treatments for OA include paracetamol (acetaminophen) as a first-line treatment, oral and topical NSAIDs as a second-line treatment and tramadol and intra-articular corticosteroid injections as third-line treatment. Opioids, duloxetine, and intra-articular hyaluronate injections are recommended when these initial treatments fail [2, 9]. Non-pharmacological modalities include biomechanical interventions, land-based and water-based exercise, strength training, and weight management. The efficacy of glucosamine sulfate, chondroitin sulphate, and avocado soybean unsaponifiables remains uncertain. Most of these treatments relieve the symptoms of OA, but they do not modify the underlying cause of the disease, chronic inflammation. Therefore, in recent years many natural compounds have been investigated, due to the minimal side effects [10-14].

Among these natural compounds polyphenols and especially curcumin gained the attention of the scientific world. Curcumin is derived from turmeric and possesses both anti-inflammatory and antioxidative activities. Buhrmann et al. proved the suppressor activity of curcumin on the activation of IKB (inhibitor of nuclear factor kappa-light-chainenhancer of activated $B$ cells [NF- $\kappa B]$ ) kinase (IKK), the phosphorylation of AKT (protein kinase $\mathrm{B}$ ) and the association between the two signaling molecules induced by IL-1 $\beta$ [33]. In turn, curcumin is able to inhibit translocation of NF- $\kappa \mathrm{B}$ into the nucleus, thus preventing the inflammatory response of the cells [33]. Seo et al. confirmed these results and showed how curcumin could also prevent the decrease of Bcl-2 and Bcl-XL expressions and the increase of Bax and caspase- 3 expression stimulated by IL-1 $\beta$. A decrease of $\mathrm{Bcl}-2 / \mathrm{Bax}$ and $\mathrm{Bcl}-\mathrm{XL} / \mathrm{Bad}$ ratio is associated with the loss of mitochondrial membrane potential [34]. On the other hand, Yan at al. highlighted that curcumin increases the phosphorylation and DNA binding activity of the nuclear factor erythroid 2-related factor 2 to the antioxidant response element [35]. This translates into a reduction of inflammation and oxidative stress in experimental animals and humans supplemented with curcumin [36]. This preliminary evidence justifies the potential of curcumin as an anti-osteoarthritic agent [36, 37].

In 2016, Chin KY. et al. carried out a thorough meta-analysis and highlighted a total of 15 relevant clinical studies on the effects of curcumin supplementation on osteoarthritic patients. All these studies demonstrated how patients with OA showed improvement in pain, physical function, and quality of life after taking curcumin. They also reported reduced concomitant usage of analgesics and reduced side effects during treatment [38].

However, while the benefits of turmeric seem to be irrefutable, some researchers consider its poor bioavailability in the human body the main drawback to using curcumin. Recently, some studies confine this poor bioavailability when used alone, highlighting how an approach to counteract this effect may be a combination treatment with several polyphenols or with polyphenols and other drugs [39-43]. Ginger, an important ingredient in Asian medicine, works synergistically with curcumin and showed anti-inflammatory effect.

Over the years, many studies have tried to understand the mechanisms behind their anti-inflammatory action, revealing how a response could be mediated in the SIRT1 pathway. SIRT1 and AMPK are two important proteins involved in many pathophysiological processes, which are able to activate each other: AMPK activates SIRT1 by increasing the Nicotinamide phosphoribosyltransferase (NAMPT) levels and SIRT1 stimulates AMPK through LKB1 deacetylation [43, 44]

SIRT1 is a NAD+ dependent histone/protein deacetylase and is able to deacetylate a lot of substrates, including p53, NF-kB, FOXO transcription factors, $\mathrm{Ku}-70$, PPAR- $\gamma$, and PGC-1 $\alpha$, with roles in cellular processes ranging from energy metabolism to cell survival [43-46].

AMPK is a fuel-sensing enzyme activated by a decrease in the cell's energy state to inhibit anabolic processes and to increase catabolic processes with the aim of restoring ATP reserves [43, 47-50]. Recent work suggests a relationship between SIRT1/AMPK, the inflammatory processes and OA, underlining how their activation could be crucial in this context and potentially protective against OA [50-54]

This study investigated the effect of a bio-optimized curcumin on the genetic (SIRT1 and AMPK) and metabolic regulation of inflammation and associated symptomatology in patients with OA.

The bio-optimized curcumin used in this study was commercialized under the brand name Curcudyn ${ }^{\circledR}$ (Metagenics) and formulated to overcome the problem of bioavailability described above. The assimilability of curcumin can be increased thanks to the use of specific technologies such as CurcuDyn's micellar technology®. This technology (NovaSol patent) helps to contain the liposoluble curcumin in a water-soluble fulcrum, guaranteeing a 185-fold increase in assimilability compared to to standard turmeric powder [55]. 
Our in vitro study shows that Curcudyn doesn't affect the viability of Hela cells as their viability remained high after $24 \mathrm{~h}$ exposure to the product. Additionally, we provide evidence that Curcudyn is able to stimulate SIRT1 $(p<0.001)$ and activate AMPK $(p<0.001)$, in support of the studies that highlight the importance of the SIRT1/AMPK pathway in many diseases, especially in OA.

The in-vivo trial showed beneficial effects of curcuma as adjuvant therapy on the health condition and an improvement in pain and quality of life. The WOMAC score analysis confirmed the SF36 results. In addition, all groups taking Curcudyn showed a significant reduction of inflammatory markers, as proof of the curcumin modulation of inflammation.

Regarding the inflammatory markers IL-1, IL-6 and IFN- $\gamma$ a significant difference between the treatment groups compared to the control group was found, confirming the anti-inflammatory effect of curcuma. Additionally, SIRT 1 expression was stimulated.The addition of vitamin D didn't show any beneficial effect to this treatment.

Limitations of this trial are the small patient population and inequal distribution of the patients in the treatment groups. As in vivo trials assessing blood parameters encounter a huge variability on the data, more patients could lead to lower standard errors and an amelioration of statistical results. Future trials should recruit more patients in order to counteract the variability, drop-out and inequal distribution of patients in all treatment groups. Future studies should also investigate the need for the standard therapy on the long term when curcuma is taken as adjuvant therapy. A dose-lowering protocol could give good perspectives for these patients.

\section{Conclusion}

In this study we tested a bio-optimized curcumin, formulated to overcome the problem of bioavailability described above. Our data demonstrate how curcuma as adjuvant to the standard treatment of osteoarthritis leads to a positive modulation of the SIRT1 pathway, a significant decline of blood inflammatory markers and a better osteoarthritis outcome.

- Osteoarthritis, a chronic degenerative disease characterized by an inflammatory state and significant oxidative stress

- Curcuma as alternative therapy next to or together with the standard treatment

- Effect of bio-optimized curcuma on genetic and metabolic regulation of inflammation

- Positive modulation of SIRT1 pathway, decline of blood inflammatory markers and a better OA outcome

\section{Conflict of Interest}

MS, GV and MVDD are employees of Metagenics Europe. The other authors declare to have no conflicts of interest

\section{Abbreviations}

AMPK Adenosine Monophosphate-activated Protein Kinase

ATP A+-adenosine triphosphate

BSA Bovine Serum Albumin

COX2 Cyclooxygenase-2

DMEM Dulbecco's Modified Eagle's Medium

DMSO Di-Methyl Sulf-Oxide

FBS Fetal Bovine Serum

FOXO Forkhead box protein $\mathrm{O}$

HRP Horse Radish Protein
IFN
Interferon
IL Interleukin
LKB1 Serine-threonine liver kinase B1
MMPs Matrix metalloproteinases
mTOR Mechanistic Target of Rapamycin
NAD+ Nicotinamide Adenine Dinucleotide

NAMPT Nicotinamide phosphoribosyltransferase

NF-KB Nuclear factor kappa-light-chain-enhancer of activated B cells

NSAIDs Nonsteroidal anti-inflammatory drug

OA Osteoarthritis

PGC1 1 Peroxisome proliferator-activated receptor gamma coactivator 1-alpha

PMSF Phenyl-Methane-Sulfonyl-Fluoride

PVDF PolyVinylidene DiFluoride

RONS Reactive Oxygen and Nitrogen Species

ROS Reactive Oxygen Species

SDS Sodium Dodecyl Sulfate

SF-36 Short Form (36) Health Survey

SIRT1 Sirtuin 1

ST Standard treatment

T-TBS Tween-Tris Buffered Saline

TNF Tumor necrosis factor

VitD Vitamin D

WOMAC Western Onstario and McMaster Universities Arthritis Index)

5-LOX 5-lipoxygenase

\section{References}

1. Castrogiovanni P, Trovato FM, Loreto C, Nsir H, Szychlinska MA, Musumeci G. Nutraceutical Supplements in the Management and Prevention of Osteoarthritis. Int J Mol Sci. 2016; 17:2042

2. Jiménez G, Cobo-Molinos J, Antich C, López-Ruiz E. Osteoarthritis: Trauma vs Disease. Adv Exp Med Biol. 2018; 1059:63-83.

3. Das SK, Farooqi A. Osteoarthritis. Best Pract Res Clin Rheumatol. 2008; 22:657-675.

4. Musumeci G, Aiello FC, Szychlinska MA, Di Rosa M, Castrogiovanni P, Mobasheri A. Osteoarthritis in the XXIst century: risk factors and behaviours that influence disease onset and progression. Int J Mol Sci. 2015; 16:6093-6112.

5. Neogi T, Zhang Y. Epidemiology of osteoarthritis. Rheum Dis Clin North Am. 2013; 39:1-19.

6. Guilak F, Nims RJ, Dicks A, Wu CL, Meulenbelt I. Osteoarthritis as a disease of the cartilage pericellular matrix. Matrix Biol. 2018; 71-72:40-50.

7. Hou A, Chen P, Tang H, et al. Cellular senescence in osteoarthritis and anti-aging strategies. Mech Ageing Dev. 2018; 175:83-87.

8. Goldring MB, Otero M. Inflammation in osteoarthritis. Curr Opin Rheumatol. 2011; 23:471-478.

9. Taruc-Uy RL, Lynch SA. Diagnosis and treatment of osteoarthritis. Prim Care. 2013; 40:821-vii. 
10. Perkins K, Sahy W, Beckett RD. Efficacy of Curcuma for Treatment of Osteoarthritis. J Evid Based Complementary Altern Med. 2017; 22:156-165.

11. Liu X, Machado GC, Eyles JP, Ravi V, Hunter DJ. Dietary supplements for treating osteoarthritis: a systematic review and meta-analysis. Br J Sports Med. 2018; 52:167-175.

12. Wang A, Leong DJ, Cardoso L, Sun HB. Nutraceuticals and osteoarthritis pain. Pharmacol Ther. 2018; 187:167-179.

13. Kuptniratsaikul V, Dajpratham P, Taechaarpornkul W, et al. Efficacy and safety of Curcuma domestica extracts compared with ibuprofen in patients with knee osteoarthritis: a multicenter study. Clin Interv Aging. 2014; 9:451-458.

14. Ross SM. Turmeric (Curcuma longa): Effects of Curcuma longa Extracts Compared with Ibuprofen for Reduction of Pain and Functional Improvement in Patients with Knee Osteoarthritis. Holist Nurs Pract. 2016; 30:183-186.

15. Kocaadam B, Şanlier N. Curcumin, an active component of turmeric (Curcuma longa), and its effects on health. Crit Rev Food Sci Nutr. 2017; 57:2889-2895.

16. Tsuda T. Curcumin as a functional food-derived factor: degradation products, metabolites, bioactivity, and future perspectives. Food Funct. 2018; 9:705-714.

17. Akuri MC, Barbalho SM, Val RM, Guiguer EL. Reflections about Osteoarthritis and Curcuma longa. Pharmacogn Rev. 2017; 11(21):8-12.

18. Fusi J, Bianchi S, Daniele S, et al. An in vitro comparative study of the antioxidant activity and SIRT1 modulation of natural compounds. Biomed Pharmacother. 2018; 101:805-819.

19. Liu YX, Wang GD, Wang X, Zhang YL, Zhang TL. Effects of TLR-2/NF- $\kappa B$ signaling pathway on the occurrence of degenerative knee osteoarthritis: an in vivo and in vitro study. Oncotarget. 2017; 8:38602-38617.

20. Johnson CI, Argyle DJ, Clements DN. In vitro models for the study of osteoarthritis. Vet J. 2016; 209:40-49.

21. Belcaro G, Cesarone MR, Dugall M, et al. Efficacy and safety of Meriva ${ }^{\circledR}$, a curcumin-phosphatidylcholine complex, during extended administration in osteoarthritis patients. Altern Med Rev. 2010; 15:337-344.

22. Tapsell LC, Hemphill I, Cobiac L, et al. Health benefits of herbs and spices: the past, the present, the future. Med J Aust. 2006; 185(S4):S1-S24.

23. Rosenbaum CC, O'Mathúna DP, Chavez M, Shields K. Antioxidants and antiinflammatory dietary supplements for osteoarthritis and rheumatoid arthritis. Altern Ther Health Med. 2010; 16:32-40.

24. Araya-Quintanilla F, Gutierrez-Espinoza H, Munoz-Yanez MJ, Sanchez-Montoya U, Lopez-Jeldes J. Effectiveness of Ginger on Pain and Function in Knee Osteoarthritis: A PRISMA Systematic Review and Meta-Analysis. Pain Physician. 2020; 23:E151E161.

25. Mozaffari-Khosravi H, Talaei B, Jalali BA, Najarzadeh A, Mozayan MR. The effect of ginger powder supplementation on insulin resistance and glycemic indices in patients with type 2 diabetes: a randomized, double-blind, placebo-controlled trial. Complement Ther Med. 2014; 22:9-16.

26. Bartels EM, Folmer VN, Bliddal H, et al. Efficacy and safety of ginger in osteoarthritis patients: a meta-analysis of randomized placebo-controlled trials. Osteoarthritis Cartilage. 2015; 23:1321.

27. Manoy P, Yuktanandana P, Tanavalee A, et al. Vitamin D Supplementation Improves Quality of Life and Physical Performance in Osteoarthritis Patients. Nutrients. 2017;9:799.

28. Rizzoli R, Stevenson JC, Bauer JM, et al. The role of dietary protein and vitamin $\mathrm{D}$ in maintaining musculoskeletal health in postmenopausal women: a consensus statement from the European Society for Clinical and Economic Aspects of Osteoporosis and Osteoarthritis (ESCEO) [published correction appears in Maturitas. 2015 Mar; 80(3):337]. Maturitas. 2014; 79:122-132.

29. Park CY. Vitamin D in the Prevention and Treatment of Osteoarthritis: From Clinical Interventions to Cellular Evidence. Nutrients. 2019; 11:243.

30. Chiu PR, Hu YC, Huang TC, et al. Vitamin C Protects Chondrocytes against Monosodium Iodoacetate-Induced Osteoarthritis by Multiple Pathways. Int J Mol Sci. 2016; 18:38.

31. Chaganti RK, Tolstykh I, Javaid MK, et al. High plasma levels of vitamin $\mathrm{C}$ and $\mathrm{E}$ are associated with incident radiographic knee osteoarthritis. Osteoarthritis Cartilage. 2014; 22:190-196.

32. Kraus VB, Blanco FJ, Englund M, Karsdal MA, Lohmander LS. Call for standardized definitions of osteoarthritis and risk stratification for clinical trials and clinical use. Osteoarthritis Cartilage. 2015; 23:1233-1241.

33. Buhrmann C, Mobasheri A, Busch F, et al. Curcumin modulates nuclear factor kappaB (NF-kappaB)-mediated inflammation in human tenocytes in vitro: role of the phosphatidylinositol 3kinase/Akt pathway. J Biol Chem. 2011; 286:28556-28566.

34. Seo SU, Woo SM, Lee HS, Kim SH, Min KJ, Kwon TK. mTORC1/2 inhibitor and curcumin induce apoptosis through lysosomal membrane permeabilization-mediated autophagy. Oncogene. 2018; 37:5205-5220.

35. Yan C, Zhang Y, Zhang X, Aa J, Wang G, Xie Y. Curcumin regulates endogenous and exogenous metabolism via Nrf2-FXRLXR pathway in NAFLD mice. Biomed Pharmacother. 2018; 105:274-281.

36. Benzer F, Kandemir FM, Ozkaraca M, Kucukler S, Caglayan C. Curcumin ameliorates doxorubicin-induced cardiotoxicity by abrogation of inflammation, apoptosis, oxidative DNA damage, and protein oxidation in rats. $\mathbf{J}$ Biochem Mol Toxicol. 2018;32:10.1002/jbt.22030.

37. Chin KY. The spice for joint inflammation: anti-inflammatory role of curcumin in treating osteoarthritis. Drug Des Devel Ther. 2016; 10:3029-3042.

38. Chin KY. The spice for joint inflammation: anti-inflammatory role of curcumin in treating osteoarthritis. Drug Des Devel Ther. 2016; 10:3029-3042.

39. Liu W, Zhai Y, Heng X, et al. Oral bioavailability of curcumin: problems and advancements. J Drug Target. 2016; 24:694-702.

40. Anand P, Kunnumakkara AB, Newman RA, Aggarwal BB. Bioavailability of curcumin: problems and promises. Mol Pharm. 2007; 4:807-818.

41. Jamwal R. Bioavailable curcumin formulations: A review of pharmacokinetic studies in healthy volunteers J Integr Med. 2019 Jul;17(4):310.

42. Aftab N, Vieira A. Antioxidant activities of curcumin and combinations of this curcuminoid with other phytochemicals. Phytother Res. 2010; 24(4):500-502.

43. Giovannini L, Bianchi S. Role of nutraceutical SIRT1 modulators in AMPK and mTOR pathway: Evidence of a synergistic effect. Nutrition. 2017; 34:82-96.

44. Narala SR, Allsopp RC, Wells TB, et al. SIRT1 acts as a nutrientsensitive growth suppressor and its loss is associated with increased AMPK and telomerase activity. Mol Biol Cell. 2008; 19:1210-9.

45. Naiman $\mathrm{S}$, Cohen HY. The contentious history of sirtuin debates. Rambam Maimonides Med Journal. 2012; 3.

46. Houtkooper RH, Pirinen E, Auwerx J. Sirtuins as regulators of metabolism and healthspan. Nat Rev Mol Cell Biol. 2012; 13:22538 . 
47. Chang HC, Guarente L. SIRT1 and other sirtuins in metabolism. Trends Endocrinol Metab. 2014; 25:138-45.

48. Hardie DG. AMPK: positive and negative regulation, and its role in whole-body energy homeostasis. Curr Opin Cell Biol. 2015; 33:1-7.

49. Shirwany NA, Zou MH. AMPK: A cellular metabolic and redox sensor. A minireview. Front Biosci .2014; 19:447-74. Review

50. Sun Y, Li J, Xiao N, et al. Pharmacological activation of AMPK ameliorates perivascular adipose/endothelial dysfunction in a manner interdependent on AMPK and SIRT1. Pharmacol Res. 2014; 89:19-28.

51. Feng K, Chen Z, Pengcheng L, Zhang S, Wang X. Quercetin attenuates oxidative stress-induced apoptosis via SIRT1/AMPKmediated inhibition of ER stress in rat chondrocytes and prevents the progression of osteoarthritis in a rat model. J Cell Physiol. 2019; 234:18192-18205.
52. Qiu L, Luo Y, Chen X. Quercetin attenuates mitochondrial dysfunction and biogenesis via upregulated AMPK/SIRT1 signaling pathway in OA rats. Biomed Pharmacother. 2018; 03:1585-1591.

53. Huang $\mathrm{H}$, Wang $\mathrm{ZJ}$, Zhang $\mathrm{HB}$, et al. The Function of PPAR $\gamma /$ AMPK/SIRT-1 Pathway in Inflammatory Response of Human Articular Chondrocytes Stimulated by Advanced Glycation End Products. Biol Pharm Bull. 2019; 42:1303-1309.

54. Liu S, Yang H, Hu B, Zhang M. Sirt1 regulates apoptosis and extracellular matrix degradation in resveratrol-treated osteoarthritis chondrocytes via the $W n t / \beta$-catenin signaling pathways. Exp Ther Med. 2017; 14(5):5057-5062.

55. Schiborr C, Kocher A, Behnam D, Jandasek J, Toelstede S, Frank $\mathrm{J}$. The oral bioavailability of curcumin from micronized powder and liquid micelles is significantly increased in healthy humans and differs between sexes. Mol. Nutr. Food Res. 2014; 58:516527. 